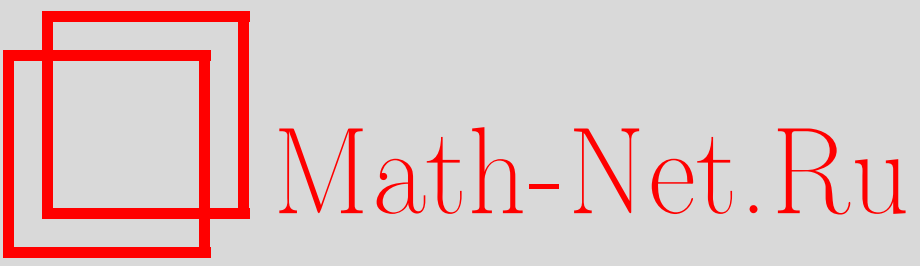

В. П. Серебряков, Об индексе дефекта матричных дифференциальных операторов второго порядка, УМН, 1997, том 52, выпуск 6, 183-184

DOI: https://doi.org/10.4213/rm913

Использование Общероссийского математического портала Math-Net.Ru подразумевает, что вы прочитали и согласны с пользовательским соглашением

http://www.mathnet.ru/rus/agreement

Параметры загрузки:

IP: 52.6 .47 .48

26 апреля 2023 г., 16:37:39 


\title{
ОБ ИНДЕКСЕ ДЕФЕКТА МАТРИЧНЫХ ДИФФЕРЕНЦИАЛЬНЫХ ОПЕРАТОРОВ ВТОРОГО ПОРЯДКА
}

\author{
В. П. СЕРЕБРяКОВ
}

Рассмотрим матричное диффференциальное выражение

$$
l[y]=-\left\{Q_{0}(x) y^{\prime}\right\}^{\prime}+Q(x) y, \quad 0 \leqslant x<\infty,
$$

где

$$
Q_{0}(x)=\left(\begin{array}{cc}
0 & q_{0}(x) \\
q_{0}(x) & 0
\end{array}\right), \quad Q(x)=\left(\begin{array}{cc}
q_{1}(x) & q(x) \\
q(x) & q_{2}(x)
\end{array}\right)
$$

суть квадратные матрицы второго порядка, элементы $q_{0}(x), q_{1}(x), q_{2}(x), q(x)$ которых есть действительнозначные функции, причем три последние из них и $\left\{q_{0}(x)\right\}^{-1}$ локально суммируемы на полуоси $I=[0, \infty)$, а $y=\left(y_{1}(x), y_{2}(x)\right)^{\tau}$ есть двухкомпонентная вектор-функция $(\tau-$ символ транспонирования).

Приведенные ниже утверждения дают соответствующие результаты относительно индекса дефекта минималшного замкнутого симметрического оператора, порождаемого выражением $l[y]$ в пространстве $L^{2}(I)$ двухкомпонентных вектор-функций (см. [1], [2], а также литературу, указанную в конце этих работ).

Наряду с выражением $l[y]$ рассмотрим более общее выражение

$$
m[y]=-\left\{P_{0}(x) y^{\prime}\right\}^{\prime}+P(x) y, \quad 0 \leqslant x<\infty ;
$$

здесь $y=\left(y_{1}(x), y_{2}(x)\right)^{\tau}$ - двухкомпонентная вектор-функция, $P_{0}(x)$ и $P(x)$ - квадратные матрицы второго порядка, $P_{0}(x)=Q_{0}(x)+i R_{0}(x), P(x)=Q(x)+i R(x)$,

$$
\begin{aligned}
& Q_{0}(x)=\left(\begin{array}{cc}
0 & q_{0}(x) \\
q_{0}(x) & 0
\end{array}\right), \quad R_{0}(x)=\left(\begin{array}{cc}
0 & r_{0}(x) \\
r_{0}(x) & 0
\end{array}\right), \\
& Q(x)=\left(\begin{array}{cc}
q_{1}(x) & q(x) \\
q(x) & q_{2}(x)
\end{array}\right), \quad R(x)=\left(\begin{array}{cc}
r_{1}(x) & r(x) \\
r(x) & r_{2}(x)
\end{array}\right),
\end{aligned}
$$

причем функции $q_{0}(x), r_{0}(x), q(x), q_{1}(x), q_{2}(x), r(x), r_{1}(x), r_{2}(x)$ действительнозначны, последние шесть из них и $\left\{p_{0}(x)\right\}^{-1}$ локалшно суммируемы на $I$, где $p_{0}(x)=q_{0}(x)+i r_{0}(x)$.

Каждое из уравнений $l[y]=0$ и $m[y]=0$ в силу наложенных на их коэффициенты условий имеет четыре линейно независимых решения. Сформулируем результаты о числе решений из пространства $L^{p}(I)$ двухкомпонентных вектор-функций этих уравнений. $L^{p}$-свойства решений скалярного уравнения Штурма-Лиувилля с действительнозначньц потенциалом при $1 \leqslant p \leqslant \infty$ изучались в работе [3] (см. также [4]), а с комплекснозначным потенциалом при $p=2$ - в [5].

Теорема 1. Пусть существует последовательность попарно непересекающихся конечных интервалов $I_{n}=\left(a_{n}, b_{n}\right) \subset I \quad(n=1,2, \ldots)$ такая, что:

1) почти всюду на $\bigcup_{n} I_{n}$ функиия $q_{0}(x)$ положстельна, а функиии $q_{1}(x)$ и $q_{2}(x)$ либо обе неотричательнь, либо обе неположительнь;

2) при некотором $p, 1<p<\infty,\left\{q_{0}(x)\right\}^{\frac{p}{1-p}}$ суммируема на каждом $I_{n}$ и ряд

расходится, где

$$
\sum_{n=1}^{\infty}\left\{\int_{I_{n}}\left\{q_{0}(x)\right\}^{\frac{p}{p-1}} d x\right\}^{1-p}\left\{\sigma\left(a_{n}, b_{n}\right)\right\}^{\frac{3 p}{2}}
$$

3) имеет место неравенство

$$
\sigma\left(x_{1}, x_{2}\right)=\int_{x_{1}}^{x_{2}}\left\{q_{0}(x)\right\}^{-1} d x
$$

$$
\int_{I_{n}} \sigma\left(a_{n}, x\right) \sigma\left(x, b_{n}\right) q_{-}(x) d x \leqslant \sigma\left(a_{n}, b_{n}\right) \quad(n=1,2, \ldots),
$$

где $q_{-}(x)=-\min \{q(x), 0\}$.

Тогда уравнение $l[y]=0$ имеет решение, не принадлежащее $L^{p}(I)$. 
ТеОРема 2. Пусть существуют последовательность попарно непересекающихся конечных интервалов $I_{n}=\left(a_{n}, b_{n}\right) \subset I$ и последовательность интервалов $I_{n}^{\prime}=$ $\left(a_{n}^{\prime}, b_{n}^{\prime}\right) \subset I_{n}(n=1,2, \ldots)$ maкuе, что:

1) для некоторого действительного $\alpha$ функция $q_{0}(x) \cos \alpha+r_{0}(x) \sin \alpha$ положительна почти всюду на $\bigcup_{n} I_{n}$ и

$$
\int_{a_{n}}^{a_{n}^{\prime}} \rho(x) d x \geqslant \gamma \int_{I_{n}} \rho(x) d x, \quad \int_{b_{n}^{\prime}}^{b_{n}} \rho(x) d x \geqslant \gamma \int_{I_{n}} \rho(x) d x \quad(n=1,2, \ldots),
$$

где $\rho(x)=\left\{q_{0}(x) \cos \alpha+r_{0}(x) \sin \alpha\right\}^{\frac{1}{2}}\left|p_{0}(x)\right|^{-1}, \gamma>0-$ постоянная;

2) функиия

$$
\begin{aligned}
\mu(x)= & q(x) \cos \alpha+r(x) \sin \alpha-\frac{1}{2}\left\{\left\{q_{1}(x)\right\}^{2}+\left\{q_{2}(x)\right\}^{2}+\left\{r_{1}(x)\right\}^{2}+\left\{r_{2}(x)\right\}^{2}\right. \\
& \left.+2\left\{q_{1}(x) q_{2}(x)-r_{1}(x) r_{2}(x)\right\} \cos 2 \alpha+2\left\{q_{1}(x) r_{2}(x)+q_{2}(x) r_{1}(x)\right\} \sin 2 \alpha\right\}^{\frac{1}{2}}
\end{aligned}
$$

неотрицательна почти всюду на $\bigcup_{n} I_{n}$ и для некоторого $p, 2 \leqslant p<\infty$, ряд

$$
\sum_{n=1}^{\infty}\left(b_{n}-a_{n}\right)^{1-\frac{p}{2}}\left\{\int_{I_{n}} \rho(x) d x\right\}^{p}\left\{\exp \left(\theta \int_{I_{n}^{\prime}} \rho(x)\{\mu(x)\}^{\frac{1}{2}} d x\right)-1\right\}^{\frac{p}{2}}
$$

расходится при некоторой постоянной $\theta, 0<\theta<1$.

Тогда уравнение $m[y]=0$ имеет не более двух линейно независимых решений из $L^{p}(I)$.

Из теоремш 2 легко получается

СлЕДСТВИЕ (cp. с [4], [6], [7]). Пусть $q_{0}(x)>0$ или $q_{0}(x)<0$ почти всюду на I и уравнение $l[y]=\lambda y$, где $\lambda-$ постоянное комплексное число, имеет более двух линейно независимых решений из $L^{p}(I)$ при некотором $p, 2 \leqslant p<\infty$. Тогда для любого $\varepsilon>0$ найдется множество $E \subset I$ положительной лебеговой мерь меньше $\varepsilon$ и локально суммируемая на I функиия $q_{\mathrm{E}}(x)$ такие, что $q_{\mathrm{E}}(x)=q(x)$ при $x \in I \backslash \mathrm{E}$, и если в $l[y]$ заменить $q(x)$ на $q_{\mathrm{E}}(x)$ (не изменяя других коэффициентов), уравнение $l[y]=\lambda y$ будет иметь не более двух линейно независимьх решений из $L^{p}(I)$; если функция $q(x)$ удовлетворяет каким-либо условиям гладкости, то функцию $q_{\mathrm{E}}(x)$ можно также выбрать из этого класса.

\section{СПИСОК ЛИТЕРАТУРЫ}

[1] Серебряков В. П. // Вестник МГУ. Сер. матем., мех. 1992. №6. С. 7-9. [2] Серебряков В.П. // Изв. вузов. Математика. 1995. № 3. С. 45-50. [3] Мирзоев К. А. // Матем. заметки. 1990. Т. 47. № 4. С. 77-82. [4] Мирзоев К. А. // Функц. анализ и его прилож. 1997. T. 31. № 1. C. 80-83. [5] Read T. T. // Lect. Notes in Math. 1983. V. 1032. P. 407-426. [6] Eastham M.S.P., Thompson M. L. // Quart. J. Math. Oxford Ser. (2). 1973. V. 24. №96. Р. 531-535. [7] Исмагилов Р. С. // УМН. 1963. Т. 18. № 5. С. 161-166. 